\title{
Positive Emotions and Self-Management Behaviors of Patients Undergoing Hemodialysis
}

\author{
Jieun $\mathrm{Cha}^{1 *}$ and Yeoungsuk Song ${ }^{2}$ \\ ${ }^{1,2}$ Assistant Professor, Kyungpook National University College of Nursing, Korea \\ 1*jecha@knu.ac.kr, ${ }^{2}$ asansong@knu.ac.kr
}

\begin{abstract}
Studies of human emotions have predominantly discussed negative emotions and negative effects, whereas positive emotions and beneficial effects have rarely been considered. The purpose of this study was to explore the potential effects of positive and negative emotions on self-management behavior among persons undergoing hemodialysis. A descriptive study and secondary analysis were conducted using two surveys that were administered two years apart (2014, 2016). A total of 250 hemodialysis patients were included in the first survey and 75 participated in the two-year follow-up survey in 2016. Participants were recruited from nine hemodialysis clinics in Seoul. Self-efficacy, serenity, and depression were measured at baseline, and self-management was assessed at the two-year follow-up. Baseline self-efficacy $(r=.36, p=.002)$ and serenity $(r=.32, p=.005)$ were positively associated with follow-up self-management behavior. Hierarchical multiple regression analysis showed baseline selfefficacy was a significant predictor of self-management behaviors, explaining $10.7 \%$ of the variance $(F=5.14, p=.003)$. This study demonstrated the importance of maintaining a positive psychological state, particularly self-efficacy and serenity, while coping with a long-term chronic disease. Improving self-efficacy may increase self-management behavior of patients undergoing hemodialysis or potentially of patients with other chronic therapies or conditions.
\end{abstract}

Keywords: Emotions, Renal dialysis, Self-efficacy, Self-management

\section{Introduction}

The incidence and prevalence of ESRD are increasing worldwide, and South Korea has also shown a rapid increase in ESRD of approximately 10\% annually since 2001 [1]. Patients with ESRD experience many medically-required demands and lifestyle changes, such as regular hemodialysis treatment 2-3 times per week, restricted fluid intake, dietary changes, and medication, and these changes require active and comprehensive self-management [2].

Self-management in context of hemodialysis as patients' positive efforts to oversee and participate in their health care to optimize their health, prevent complications, control symptoms, marshal medical resources, and minimize the intrusion of the disease into their preferred lifestyle [2]. Thus, self-management involves more than simply making changes in disease-related behavior; it also involves adapting to and coping with changes in lifestyle as a result of disease.

Optimal self-management must be supported by self-control [3]. Self-control is a highly adaptive and important ego function, and proper self-control requires psychological resources [4]. In previous research, positive emotions were found to supply resources for self-control [5].

Article history:

Received (December 10, 2020), Review Result (January 13, 2021), Accepted (February 20, 2021) 
Positive emotions, which restore ego and strengthen resources after depletion, can improve this situation. Positive emotions are expected to be a useful resource for self-management behaviors [6].

In the field of positive psychology, empirical findings have demonstrated the salutary functions of positive emotions in humans, grounded in theories that support their adaptive functions [5]. One notable theory is the "Broaden-and-Build Theory of Positive Emotions", in which positive emotions lead to long-term growth and progress by broadening thoughts and behaviors and building resources. However, theoretical models are valuable and useful in developing effective interventions only when they are based on empirical research. Therefore, it is necessary to empirically investigate the relationship between positive emotions and selfmanagement behaviors.

Individuals who experienced a high level of positive emotions have been found to be more successful in coping throughout the course of their disease [7]. Self-efficacy is the belief that one can successfully resolve a certain problem by one's own ability, which helps individuals maintain their behaviors in the face of difficulties and to recover after failures. Serenity is a sense of peace and tranquility as opposed to anxiety and confusion, and it forms the basis of a happy mind [8]. This emotional experience of inner peace is sustained regardless of life events and implies harmony of body, spirit, and mind. In contrast, depression is a negative emotion commonly experienced by hemodialysis patients and a risk factor negatively affecting selfmanagement [9].

Thus far, studies of human emotions have predominantly discussed negative emotions and negative effects, whereas positive emotions and beneficial effects have rarely been considered [10]. However, few existing studies have analyzed the effects of positive emotions on selfmanagement behaviors of patients with chronic disease. Therefore, this study focuses on selfefficacy and serenity as positive emotion experienced during the course of hemodialysis patients' disease, aiming to test their association with self-management behaviors.

\section{Methods}

\subsection{Study design}

This study uses a descriptive design which aimed to examine the relationship between positive emotions and self-management behaviors of patients undergoing hemodialysis.

\subsection{Participants}

This investigation used secondary data from two surveys within a period of two-year. A total of 250 hemodialysis patients were included in the first survey, conducted in January 2014 [11]; of these, 75 participated in the two-year follow-up survey around January 2016 [12]. Participants were recruited from nine hemodialysis clinics in Seoul.

\subsection{Measurements}

1) Self-efficacy

Self-efficacy refers to belief in one's ability to succeed in specific situations or to accomplish specific tasks. The original instrument to measure self-efficacy, developed by Sherer et al. [13], consists of 17 items measured on a 5-point scale. The present study employed modified version of Shere et al.'s scale for Korean patients receiving hemodialysis [14], which included 7 items measured on a 4-point scale from 0 (strongly disagree) to 3 (strongly agree), for a total score 
ranging from 0 to 21 . Higher scores indicate stronger self-efficacy. Cronbach's alpha in the present study was .82 .

2) Serenity

Serenity is a low-intensity positive emotion that reflects human integration and peacefulness. Self-reported serenity among hemodialysis patients was assessed using one item similar in meaning to "positive affect" from the psychological health problem items of the quality-of-life instrument in the Short Form Health Survey (SF-12): "Have you felt calm and peaceful during the past four weeks?" Responses were recorded on a 5-point Likert scale from 1 (all the time) to 5 (none of the time). The score (1-5) was reversed and converted into a score from 0 to 100. The higher the score, the more serene the participant.

3) Depression

Depression was measured using the Hospital Anxiety and Depression Scale (HADS). The HADS comprises two subscales with seven items each for anxiety and depression; the seven depression items were adopted in this study. Items were scored on a 4-point Likert scale from 0 (not at all) to 3 (often), for a total score ranging from 0 to 21. Scores of 0-7 are considered normal; scores of 8-10 are considered borderline; and scores of 11 or higher indicate probable presence of depression. Cronbach's alpha was .79 in current study.

4) Self-management behavior

The Hemodialysis Self-Management Instrument [15] was used to measure self-management behavior. This scale has 20 items in four subscales: problem solving (5 items), partnership (4 items), self-care (7 items), and emotional management (4 items). Each item is scored on a 4point scale from 1 (never) to 4 (always). Total scores range from 20 to 80, with higher scores reflecting higher levels of self-management. Cronbach's alpha was .91 for the present study.

\subsection{Data collection and procedures}

The Institutional Review Board of K University approved this study as a secondary analysis of data. Original data were collected in two ways: through in-person visits to the hemodialysis clinic by a researcher, and/or through online support groups for hemodialysis patients. The researcher explained the purpose and content of the study, and then obtained informed consent from patients who agreed to participate in the study. Positive (self-efficacy and serenity) and negative (depression) variables were measured at baseline (T1), and self-management was measured at follow-up (T2) [Figure 1].

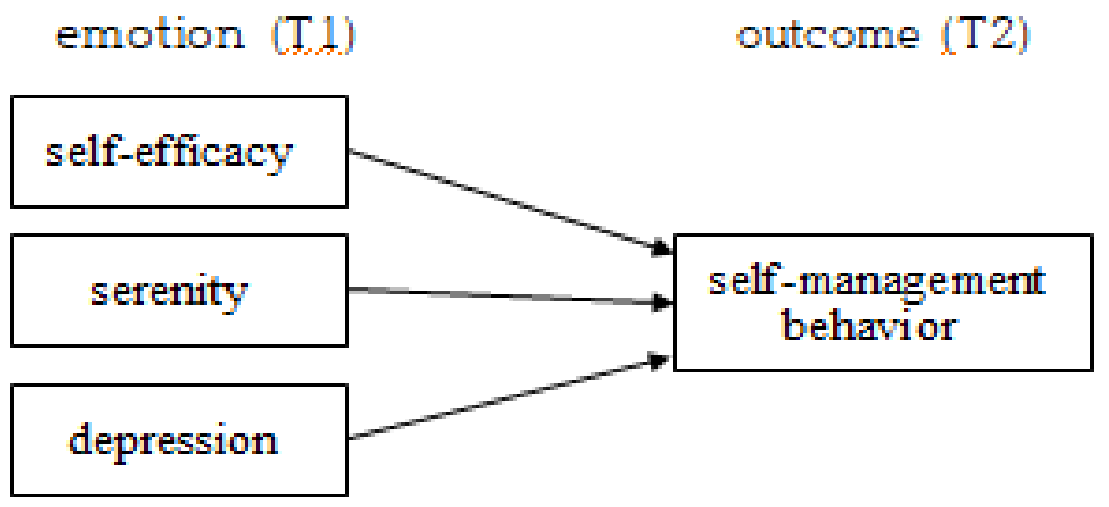

Figure 1. Schematic diagram 


\subsection{Data analysis}

IBM SPSS Statistics 23 was used to analyze the data. Participants' characteristics, emotions and self-management behavior were analyzed using descriptive statistics. Independent $t$-tests and analysis of variance (ANOVA) were used to examine self-management behavior by general characteristics. Pearson correlation coefficients were used to examine bivariate associations among baseline assessments of self-efficacy, serenity, depression, and self-management measured at follow-up. Hierarchical multiple regression analysis was used to identify predictors of self-management. All significance levels were set at $\alpha=.05$.

\section{Results}

\subsection{Demographic and clinical characteristics}

The mean age of participants was 47.3 , and the majority were male $(68 \%)$. The majority were married (62.7\%) and had an education level of high school or lower $(62.7 \%) ; 52.0 \%$ had a monthly income above 2 million won; $42.7 \%$ were employed; and $62.7 \%$ reported they had a specific religion. Mean years on hemodialysis was 8.3. Hypertension was the most common primary disease (44.0\%), followed by glomerulonephritis (30.7\%). $40.0 \%$ of participants were waiting for kidney transplantation.

\subsection{The degree of self-efficacy, serenity, depression and self-management behavior}

Mean scores of self-efficacy and serenity were 12.5 and 55.7, respectively. The depression mean score was 6.7 , with $51.7 \%$ participants classified as non-depressed, $32.0 \%$ as borderline, and $13.3 \%$ as probable cases of depression. The mean total self-management behavior score at the two-year follow-up was 64.1. Problem-solving was the highest self-management behavior subscale item with 17.1 points, followed by self-care (23.0), partnership (12.6), and emotional management (11.4).

\subsection{Relationships of self-efficacy, serenity, depression and self-management behavior}

In bivariate analyses at baseline, self-efficacy and serenity were negatively correlated with depression $(\mathrm{r}=-.25, p=.031 ; \mathrm{r}=-.55, p<.001)$, and self-efficacy was positively associated with serenity $(r=.36, p=.002)$. Further, baseline self-efficacy $(\mathrm{r}=.36, p=.002)$ and serenity $(\mathrm{r}=.32, p=.005)$ were positively associated with follow-up self-management behavior, and depression was negatively associated with follow-up self-management behavior $(\mathrm{r}=-.27, p=$ $.021)$

\subsection{Predictors of self-management behavior}

In multivariate analyses, the variance inflation factor (VIF), used to check for multicollinearity, was $1.15-1.54$, indicating no serious multicollinearity problems among the predictor variables. The Durbin-Watson value was 1.81, indicating that the residuals from linear regression were uncorrelated. Depression was entered in Model 1 and accounted for $7.1 \%$ of the variance in self-management behavior at follow-up $(\mathrm{F}=5.56, p=.021)$. The two positively oriented measures (self-efficacy and serenity) were entered in Model 2. These variables explained $10.7 \%$ of the variance $(\mathrm{F}=5.14, p=.003)$; baseline self-efficacy $(\beta=.27, p=.021)$ was a significant predictor of follow-up self-management behavior.

\section{Discussion}


An analysis of the effects of positive emotions on self-management in hemodialysis patients was conducted and a significant relationship was found between positive emotions and selfmanagement behavior. This indicates that in addition to controlling negative emotions like depression, reinforcing positive factors, such as self-efficacy and serenity, is also important in improving self-management behavior.

Level of self-efficacy and serenity in this study were $12.5 / 21$ and 55.7/100, separately in hemodialysis patients, demonstrating that despite the difficulties caused by chronic disease, patients were able to experience a positive psychological state in the process of coping with their disease. Thirty-four patients (45.3\%) demonstrated a clinically significant score of 8 or higher for depression as a negative emotion, demonstrating the co-occurrence of the negative as well as positive emotions and beliefs in stressful circumstances. Our study found that hemodialysis patients experience positive emotions and beliefs (serenity and self-efficacy) amidst the constant stress of chronic disease, providing empirical evidence for the adaptive value of positive emotions and beliefs.

The participants in this study exhibited $64.1 / 80$ points of self-management behavior, and among the subdomains of self-management behavior, problem-solving was the highest. We interpret this to mean that this is potentially a result of the high proportion of long-term dialysis patients in the sample, whose problem-solving ability had grown through a process of trial and error during the long period of their disease.

Hemodialysis patients who initially showed higher self-efficacy and serenity at baseline showed better performance of self-management behaviors at two-year follow-up. Similar results have been reported in patients with other chronic diseases, such as diabetes [16]. Higher perceived competence and motivation in diabetes patients was associated with higher levels of self-management, and at a one-year follow-up, these patients showed better control of blood glucose levels [16]. Thus, considering the functions of positive emotions such as serenity, as well as positive beliefs of one's self-efficacy, one might expect adaptive coping behaviors, such as self-management, during the course of disease.

The present study showed that higher self-efficacy was associated with better ability to cope with the various demands of disease. High self-belief and personal expectations allow patients to focus on the tasks at hand and to actively use coping strategies to effectively solve problems. Additionally, hemodialysis patients with higher levels of mental stability and peace (serenity) showed higher likelihood of performing self-management behaviors. Serenity is a relaxed positive emotion equivalent to "peace of mind," which indicates internal peace and harmony in Eastern cultures, including those of Korea, China, and India [8]. Our study demonstrated for the first time that maintaining a stable, calm mental state had a positive effect on selfmanagement behaviors in patients with chronic disease.

When the negative emotion of depression was added to the regression model to analyze the effects of emotions on self-management two years later, self-management behaviors decreased significantly, and explanatory power was 7.1\%. Similarly, a study by Li et al., [15] in China, using the same instruments as the present study, also found that depression affected selfmanagement behaviors. Depressed patients could not escape their negative thoughts, lacked cognitive flexibility, and demonstrated limitations in various coping strategies [9]. However, when the positive factors of self-efficacy and serenity were added to the regression model, the effects of depression disappeared seemingly due to the statistical significance of self-efficacy.

This study demonstrated that positive factors, such as self-efficacy and serenity, can promote self-management behaviors. Although an increasing number of studies have identified the functions of positive states, most have focused on healthy subjects. In this study, we used data from hemodialysis patients to provide empirical evidence of the effects of positive emotions in 
the context of health care and disease. Although preliminary, the results of this study are valuable in that they show that the positive emotions of self-efficacy and serenity were predictive of health-related behaviors of self-management among patients with chronic disease.

There are some limitations to the present study. Because serenity was measured by only a single question, subsequent studies will need to improve validity by using more multidimensional scales. In addition, since self-management behavior was not measured at baseline, the statistical conclusion validity is threatened. In addition, the sample size of the study was relatively small, and we were able to conduct only a single follow-up survey regarding self-management behaviors. Therefore, it will be important to conduct a larger-scale study with repeated measurements to demonstrate the long-term effects of positive emotions.

\section{Conclusions}

This study demonstrated that positive factors, such as self-efficacy and serenity, can promote self-management behaviors. Results of this study showed depression, self-efficacy, and serenity affect self-management. Although preliminary, the results of this study are valuable in that they show that the positive emotions of self-efficacy and serenity were predictive of healthrelated behaviors of self-management among patients with chronic disease. It will be important for clinical nurses and family members to create a supportive environment that enables patients to experience positive emotions, and to instruct patients with chronic disease in methods to improve self-efficacy and serenity.

\section{References}

[1] http://www.ksn.or.kr/rang_board/list.html?code=sinchart, Jan 20, (2020)

[2] S. W. Ong, S. V. Jassal, E. Porter, A. G. Logan, and J. A. Miller, "Using an electronic self-management tool to support patients with chronic kidney disease (CKD): A CKD clinic self-care model," Seminars in Dialysis, vol.26, no.2, pp.195-202, (2013)

[3] M. Novak, L. Costantini, S. Schneider, and H. Beanlands, "Approaches to self-management in chronic illness," Seminars in Dialysis, vol.26, no.2, pp.188-194, (2013)

[4] M. Inzlicht and B. J. Schmeichel, "What is ego depletion? Toward a mechanistic revision of the resource model of self-control," Perspectives on Psychological Science, vol.7, no.5, pp.450-463, (2012)

[5] M. S. Hagger, C. Wood, C. Stiff, and N. L. Chatzisarantis, "Ego depletion and the strength model of self-control: A meta-analysis," Psychological bulletin, vol.136, no.4, pp.495-525, (2010)

[6] R. Moss-Morris, "Adjusting to chronic illness: Time for a unified theory,” British Journal of Health Psychology, vol.18, no.4, pp.681-686, (2013)

[7] S. D. Pressman, B. N. Jenkins, and J. T. Moskowitz, "Positive affect and health: What do we know and where next should we go?” Annual Review of Psychology, vol.70, pp.627-650, (2019)

[8] Y. C. Lee, Y. C. Lin, C. L. Huang, and B. L. Fredrickson, "The construct and measurement of peace of mind," Journal of Happiness Studies, vol.14, no.2, pp.571-590, (2013)

[9] A. A. Khali, T. A. Lennie, and S. K. Frazier, "Understanding the negative effects of depressive symptoms in patients with ESRD receiving hemodialysis,” Nephrology Nursing Journal, vol.37, no.3, pp.289-296, (2010)

[10] S. Folkman, “Stress, coping, and hope,” Psycho-oncology, vol.19, no.9, pp.901-905, (2010)

[11] J. Cha, "Structural equation modeling of quality of life focused on the self-management process in patients with hemodialysis," Ph. D. dissertation, College of Nursing, Seoul National University, Seoul, (2014)

[12] J. Cha, "Predictors of health status in patients with hemodialysis: A two-year longitudinal study," Journal of Korean Clinical Nursing Research, vol.22, no.3, pp.359-367, (2016)

[13] M. Sherer, J. E. Maddux, B. Mercandante, S. Prentice-Dunn, B. Jacobs, and R. W. Rogers, "The self-efficacy scale: Construction and validation,” Psychological Reports, vol.51, no.2, pp.663-671, (1982) 
[14] J. H. Kim, "An effect of guided imagery applied to hemodialysis patients," Ph. D. Dissertation, College of Nursing, Seoul National University, Seoul, (1995)

[15] H. Li, Y. F. Jiang, and C. C. Lin, "Factors associated with self-management by people undergoing hemodialysis: A descriptive study," International Journal of Nursing Studies, vol.51, no.2, pp.208-216, (2014)

[16] S. M. Robertson, M. A. Stanley, J. A. Cully, and A. D. Naik, "Positive emotional health and diabetes care: Concepts, measurement, and clinical implications," Psychosomatics, vol.53, no.1, pp.1-12, (2012) 
Positive Emotions and Self-Management Behaviors of Patients Undergoing Hemodialysis

This page is empty by intention. 